\title{
Specificity and Mechanism of Mandelamide Hydrolase Catalysis
}

S.A. Adediran, ${ }^{\mathrm{a}}$ Pan-Fen Wang, ${ }^{\mathrm{b}}$ Abbas G. Shilabin, ${ }^{\mathrm{c}}$ Charles A. Baron, ${ }^{\mathrm{a}}$ Michael J. McLeish,,${ }^{\mathrm{b}}$ R.F.Pratt ${ }^{\mathrm{a}, *}$

a Department of Chemistry, Wesleyan University, Middletown, CT 06459, USA

${ }^{b}$ College of Pharmacy, University of Michigan, Ann Arbor, MI 48105, USA

${ }^{c}$ Department of Chemistry, East Tennessee State University, Johnson City, TN, 37614, USA

${ }^{d}$ Department of Chemistry and Chemical Biology, Indiana University-Purdue University

Indianapolis, Indianapolis, IN 46202, USA

* Corresponding author.

Email address: rpratt@wesleyan.edu

Keywords

Mandelamide hydrolase

Amide signature enzymes

Substrates and inhibitors

Structure/activity

Inverse acyl-enzyme 


\begin{abstract}
The best-studied amidase signature (AS) enzyme is probably fatty acid amide hydrolase (FAAH). Closely related to FAAH is mandelamide hydrolase (MAH), whose substrate specificity and mechanism of catalysis are described in this paper. First, we developed a convenient chromogenic substrate, 4-nitrophenylacetamide, for MAH. The lack of reactivity of MAH with the corresponding ethyl ester confirmed the very limited size of the MAH leaving group site. The reactivity of MAH with 4-nitrophenyl acetate and methyl 4-nitrophenyl carbonate, therefore, suggested formation of an "inverse" acyl-enzyme where the small acylgroup occupies the normal leaving group site. We have interpreted the specificity of MAH for phenylacetamide substrates and small leaving groups in terms of its active site structure, using a homology model based on a FAAH crystal structure. The relevant structural elements were compared with those of FAAH. Phenylmethylboronic acid is a potent inhibitor of $\mathrm{MAH}\left(\mathrm{K}_{\mathrm{i}}=27\right.$ $\mathrm{nM}$ ), presumably because it forms a transition state analogue structure with the enzyme. O-Acyl hydroxamates were not irreversible inactivators of MAH but some were found to be transient inhibitors.
\end{abstract}




\section{Introduction}

Mandelamide hydrolase from Pseudomonas putida (MAH, EC 3.5.1.86) catalyzes the hydrolysis of mandelamide to mandelate and ammonia. MAH appears to be associated with the mandelate metabolic pathway of Pseudomonas putida, a pathway that allows this organism to utilize either of the enantiomers of mandelate as the sole carbon source. The gene encoding MAH, located within the gene cluster of the mandelate pathway enzymes of Pseudomonas putida ATCC 12633, has been identified and cloned [1]. MAH belongs to a large group of enzymes termed the amidase signature (AS) family. The AS enzymes share a highly conserved serine- and glycine rich stretch of amino acid sequence of approximately 50 amino acids. Proteins with AS sequences have been found from a wide range of organisms including archaea, eubacteria, fungi, nematodes, plants, insects, birds and mammals, where they perform diverse biological functions $[2,3]$.

The AS enzymes are different from the large group of serine hydrolases that utilize a catalytic triad of Ser-His-Asp for hydrolysis of amides or esters, and possess a unique, highly conserved Ser-Ser-Lys catalytic triad [2,3]. The mammalian enzyme fatty acid amide hydrolase (FAAH) represents one of the best characterized AS enzymes. A catalytic mechanism for FAAH has been proposed based on site-directed mutagenesis, affinity labeling, steady-state kinetics, and crystal structures [4-6]. Evidence suggests that a double displacement acyl transfer mechanism obtains with an acyl-enzyme intermediate located on Ser241. The kinetics of turnover of substrate analogues suggests that acyl-enzyme formation is generally rate limiting under substrate saturation conditions [4]. The catalytic triad of FAAH is composed of Ser241-Ser217Lys142. The proposed mechanism involves the Lys142 amine, initially in the deprotonated state, functioning as a base to activate the nucleophilic Ser241, and as an acid to facilitate the protonation of the substrate leaving group. Both functions were proposed to be achieved via the 
bridging residue Ser217. The latter intervention was thought to explain the unusual ability of FAAH to hydrolyze amides and esters at equivalent rates. The AS enzymes also appear to have active site and mechanistic similarities to other serine/amine enzymes, the $\beta$-lactam-recognizing enzymes and the N-terminal nucleophile (Ntn) amidohydrolases, probably as a result of convergent evolution $[7,8]$.

In previous studies, MAH from Pseudomonas putida has been cloned, expressed in $E$. coli and characterized [1]. The catalytic triad of MAH is composed of Ser204, Ser180, and Lys100. Mutation of the catalytic residues resulted in total loss of activity for S204A and K100A and a 4200-fold decrease in the $\mathrm{k}_{\mathrm{cat}} / \mathrm{K}_{\mathrm{m}}$ value for S180A [9]. Both (R)- and (S)-mandelamide are excellent substrates for MAH, while the enzyme can hydrolyze phenylacetamide with even higher catalytic efficiency. Kinetic data with alternative substrates indicates that the active site is sterically hindered, and the enzyme can tolerate very little substitution on the amide nitrogen. Similar to FAAH, MAH has the uncommon ability to hydrolyze esters and amides at similar rates. Here, we report the results of our studies with some new MAH substrates. We also interpret the substrate specificity structurally and examine the reactivity of MAH with some recently studied $\beta$-lactamase inhibitors.

\section{Materials and Methods}

\subsection{Materials}

Site-directed mutagenesis The MAH variants, F150L, F433A and F433L were prepared using PfuUltra HF DNA polymerase (Agilent) and the QuikChange site-directed mutagenesis protocol (Stratagene) with pET17MAH-His [1] as the template. The forward primers used for the mutagenesis are shown below with the mutated codon underlined and the lowercase letters indicating base change from the wt,

F150L: 5'- CATGAGCTAGCGcTTGGgACCTCGGGC-3'

F433A: 5'- CCAGTTTCGAGGCCgcCGCtCGCATGATTCG-3' 


\section{F433L: 5'- CCAGTTTCGAGGCCTTgGCACGCATGATTCG-3'}

The primers also introduced AvaII (F150L), BsrB1(F433A) and StyI (F433L) sites which were used for restriction analysis in initial screening for mutations. The fidelity of the PCR amplification and the presence of the desired mutations were verified by sequencing (University of Michigan Sequencing Core).

Protein expression and purification Wild-type and variant MAH were expressed with a Cterminal 6x his-tag using the previously described methods [1,9]. Following purification the enzyme was kept at $-20^{\circ} \mathrm{C}$ in a storage buffer containing $100 \mathrm{mM}$ potassium phosphate buffer, pH 7.8, containing 1 mM EDTA. Enzyme concentrations were determined by UV absorption using an $\varepsilon_{278}$ of $28,800 \mathrm{M}^{-1} \mathrm{~cm}^{-1}$ [9].

Phenylacetamide (3) (TCI), 4-nitrophenyl acetate (8) (Sigma-Aldrich), 4-nitrophenyl nbutyrate (12) (Santa Cruz Biotechnology), 4-nitroacetanilide (10) (Acros), Nbenzyloxycarbonylglycinamide (20) (Chem Impex), and phenyl carbamate (21) (Sigma-Aldrich), and methyl mandelate (7) (Sigma) were obtained from commercial sources and used as supplied. R-Mandelamide was prepared as previously described [1]. Methyl 4-nitrophenylcarbonate (11) was prepared from reaction of 4-nitrophenyl chloroformate with methanol in the presence of triethylamine and recrystallized from an ethyl acetate/hexane mixture (7:3). Ethyl 4-nitrophenyl carbonate (13) was prepared from reaction of 4-nitrophenol with ethyl choroformate in the presence of aqueous sodium hydroxide and recrystallized from ethanol [10]. Methyl (5) and ethyl (6) 4-nitrophenylacetate were obtained by reaction of 4-nitrophenylacetic acid (Acros) with methyl and ethyl chloroformate, respectively, and the products recrystallized from 2-propanol. 4Nitrophenylacetamide (4) was obtained by ammonolysis of the ethyl ester in $30 \%$ aqueous $\mathrm{NH}_{3}$ at $100^{\circ} \mathrm{C}$. On cooling of the reaction mixture, pure crystals of $\mathbf{4}$ precipitated. Compound $\mathbf{1 6}$ was previously prepared in this laboratory [11,12]. The carbamates $\mathbf{1 6}$ and $\mathbf{1 9}$ were obtained by reaction of the corresponding hydroxamic acids with trimethylsilylisocyanate (Acros) and 
purified by recrystallization from aqueous ethanol. Phenylmethylboronic acid was prepared in situ by hydrolysis of the pinacol ester (AlfaAesar).

\subsection{Methods}

All kinetics measurements with MAH were carried out at $25^{\circ} \mathrm{C}$ in $0.1 \mathrm{M}$ phosphate buffer containing $1 \mathrm{mM}$ EDTA at $\mathrm{pH}$ 7.8. The hydrolysis, ammonolysis and methanolysis of the amide 4 and the ester 5 were monitored spectrophotometrically at $310 \mathrm{~nm}\left(\Delta \varepsilon=2140 \mathrm{M}^{-1} \mathrm{~cm}^{-1}\right)$ and $320 \mathrm{~nm}\left(\Delta \varepsilon=1600 \mathrm{M}^{-1} \mathrm{~cm}^{-1}\right)$, respectively. DMSO (5\%) was included to facilitate dissolution of these substrates and MAH concentrations were $0.014 \mu \mathrm{M}$ and $0.047 \mu \mathrm{M}$ in studies of $\mathbf{4}$ and $\mathbf{5}$, repectively. Initial rates of MAH-catalyzed reactions were fitted to the Michaelis-Menten equation by a non-linear least squares method (Kaleidograph). Steady-state parameters for the MAH variants were obtained using S-mandelamide as substrate under the conditions described above.

Ammonium chloride and methanol inhibited the reaction of MAH with 4 (200 $\mu \mathrm{M}$ and 800 $\mu \mathrm{M})$ and $\mathbf{5}(1.0 \mathrm{mM})$, respectively. Under these substrate saturation conditions, the steady state equation for Scheme 3 yields equation 1 , where $\mathrm{v}$ and $\mathrm{v}_{0}$ are the initial rates in

$$
\mathrm{v}_{0} / \mathrm{v}=\left(1+[\mathrm{XH}] / \mathrm{K}_{\mathrm{i}}\right)^{-1}
$$

the presence and absence of the nucleophile $\mathrm{XH}$, respectively, and the apparent inhibition constant $\mathrm{K}_{\mathrm{i}}=\left(\mathrm{k}_{2}+\mathrm{k}_{3}\right) / \mathrm{k}_{-2}$. Thus values of $\mathrm{K}_{\mathrm{i}}$ for ammonium and methanol were obtained for 4 and $\mathbf{5}$, respectively. Initial rates of hydrolysis of $\mathbf{4}$ in the presence of several concentrations of phenylmethyl boronic acid were also obtained. These data were treated by assuming the boronic acid was a competitive inhibitor.

The temperature effect on the hydrolysis of R-mandelamide was measured using the coupled enzyme assay for ammonia [1,9] in $100 \mathrm{mM}$ phosphate buffer at $\mathrm{pH} 7.8$ and containing $1 \mathrm{mM}$ EDTA. The assay mixture comprised the substrate, mandelamide, $(10-200 \mu \mathrm{M}), 250 \mu \mathrm{M}$ 
NADH, $800 \mu \mathrm{M} \alpha$-ketoglutarate (Sigma), $15 \mathrm{U} / \mathrm{mL}$ glutamate dehydrogenase (Sigma), and MAH $(0.14-0.91 \mu \mathrm{M})$.

The temperature effect on the hydrolysis of methyl mandelate was measured using a $\mathrm{pH}$ stat assay [1] with an end point of $\mathrm{pH} 8.5$. The assay mixture comprised the substrate methyl mandelate $(100-800 \mu \mathrm{M}), 1 \mathrm{mg} / \mathrm{ml} \mathrm{BSA}$, and MAH $(9.1-36.5 \mu \mathrm{M})$. The reaction mixture was titrated with $5 \mathrm{mM}$ sodium hydroxide solution.

The hydrolysis of p-nitrophenyl acetate (8) was also studied spectrophotometrically. In a typical reaction, $5-20 \mu \mathrm{L}$ of $\mathbf{8}$ in DMSO was added to phosphate buffer $(50 \mathrm{mM}, 1.0 \mathrm{~mL})$ at $\mathrm{pH}$ 7.8. To maintain substrate solubility, DMSO was included to a final concentration of $5 \%(\mathrm{v} / \mathrm{v})$. The reaction mixture then remained transparent during the course of the measurements. After the reaction solution had reached thermal equilibrium, an aliquot of enzyme stock was added to initiate the reaction. The hydrolysis was followed spectrophotometrically by monitoring the absorbance increase at $400 \mathrm{~nm}$ due to production of p-nitrophenolate. A molar extinction coefficient of $18300 \mathrm{M}^{-1} \mathrm{~cm}^{-1}$ for $\mathrm{p}$-nitrophenolate and a $\mathrm{pK}_{\mathrm{a}}$ of 7.1 for $\mathrm{p}$-nitrophenol were assumed for calculation of the rates of hydrolysis. The observed absorbance increase at $400 \mathrm{~nm}$ represents a sum of two reactions, enzymatic hydrolysis and nonenzymatic hydrolysis of $\mathbf{8}$. Therefore, corrections were made for the nonenzymatic hydrolysis by subtracting the rate observed in the absence of enzyme from that in the presence of enzyme under the same conditions. For the determination of $k_{\text {cat }}$ and $K_{m}$ parameters, initial rate measurements were performed under steady state conditions with the substrate concentration in the range of 0.1 to $1.6 \mathrm{mM}$ and the enzyme at $45 \mathrm{nM}$. The hydrolysis of $\mathbf{9}$ and $\mathbf{1 1}-\mathbf{1 3}$ were studied in a similar fashion.

Compounds 16, 17, 19 - 21 were first tested as substrates of MAH from spectrophotometric progress curves of their reactions in the presence of the enzyme. They were 
also tested as inactivators of the enzyme by incubating each one of them separately with the enzyme and assaying aliquots of the reaction mixtures against $500 \mu \mathrm{M}$ 4-nitrophenyl acetate at $400 \mathrm{~nm}$. The initial rates, proportional to enzyme activity, were plotted against time to obtain progress curves that were fit to Scheme 1 (where Q is the hydrolysis product of inhibitor I) by the Dynafit program [13] to obtain the rate constants of inactivation. For compound 17, the inhibition of the enzyme was observed in total progress curves of the hydrolysis of $\mathbf{4}$ in the presence of the inhibitor (from adding the enzyme to a mixture of substrate and inhibitor). The resulting progress curves were fitted to Scheme 2 to obtain the kinetic parameters. The presence of MAH-catalyzed hydrolysis of $\mathbf{4 , 1 6}$, and 19 was also confirmed by means of a wellestablished ammonia assay $[1,9]$.

\section{Scheme 1}

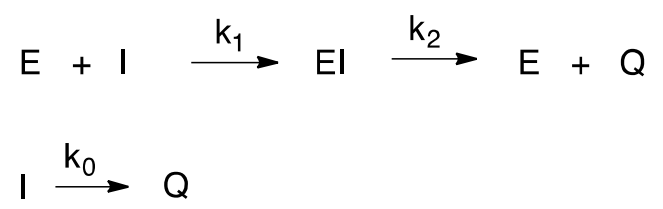

\section{Scheme 2}

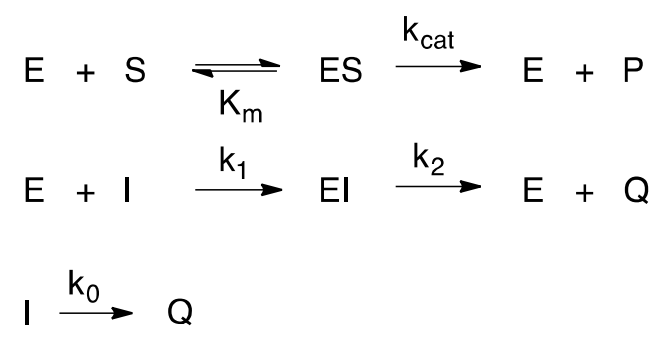

Molecular modeling simulations were performed on a SGI workstation running the program Insight II, essentially as previously described [12]. The homology structure of MAH [9] was modified to obtain the adduct $\mathbf{1 4}$ with the active site Ser 204. The ligand was placed manually following the example of the crystal structure of FAAH covalently bound through its active site to a phosphonate transition state analogue (PDB ID 1MT5 [6]). The pH was set to 7.0 where the side chain of Lys100 was cationic. The active site was hydrated with a $20 \AA$ sphere of 
water centered on $\mathrm{O} \gamma$ of the nucleophilic serine 204. The model was then subjected to 1000 steps of steepest descent energy minimization, followed by 2000 steps of conjugate gradients.

\section{Results and Discussion}

\subsection{Substrate Specificity}

Previous studies of MAH have shown that R- (1) and S-mandelamide (2) are comparably efficient $\left(\mathrm{k}_{\text {cat }} / \mathrm{K}_{\mathrm{m}}\right)$ as substrates and that phenylacetamide (3), lacking an $\alpha$-substituent, is even more efficient (Table 1) [1,9]. None of the previously described substrates, however, are suitable for a convenient direct spectrophotometric assay. The closest approximation to a direct assay is a coupled enzyme procedure using glutamate dehydrogenase to monitor ammonia released by MAH [9]. Thus, we prepared p-nitrophenylacetamide 4 and showed it to have spectrophotometric properties suitable for a direct assay (Figure 1). A ${ }^{1} \mathrm{H}$ NMR experiment showed that $\mathbf{4}$ is a MAH substrate, yielding p-nitrophenylacetate. The ammonia assay also showed release of ammonia as a product. We therefore used $\mathbf{4}$ as a convenient assay substrate for further studies of the enzyme (see below), monitoring its hydrolysis catalyzed by MAH at 310 $\mathrm{nm}\left(\Delta \varepsilon=2140 \mathrm{M}^{-1} \mathrm{~cm}^{-1}\right)$. Thus, from initial rate measurements, the steady state parameters for turnover of 4 by MAH were determined (Table 1). The methyl (5) and ethyl (6) ester of pnitrophenylacetic acid were also prepared.

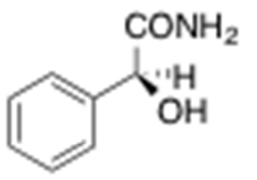

$1(R)$<smiles>NC(=O)C(O)c1ccccc1</smiles>

2(S)

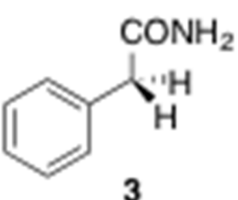

3
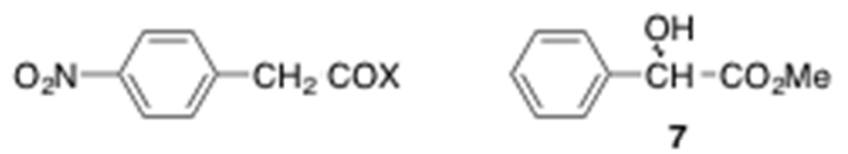

$4 \mathrm{X}=\mathrm{NH} 2$

$5 \mathrm{X}=\mathrm{OMe}$

$6 \mathrm{X}=\mathrm{OEt}$ 


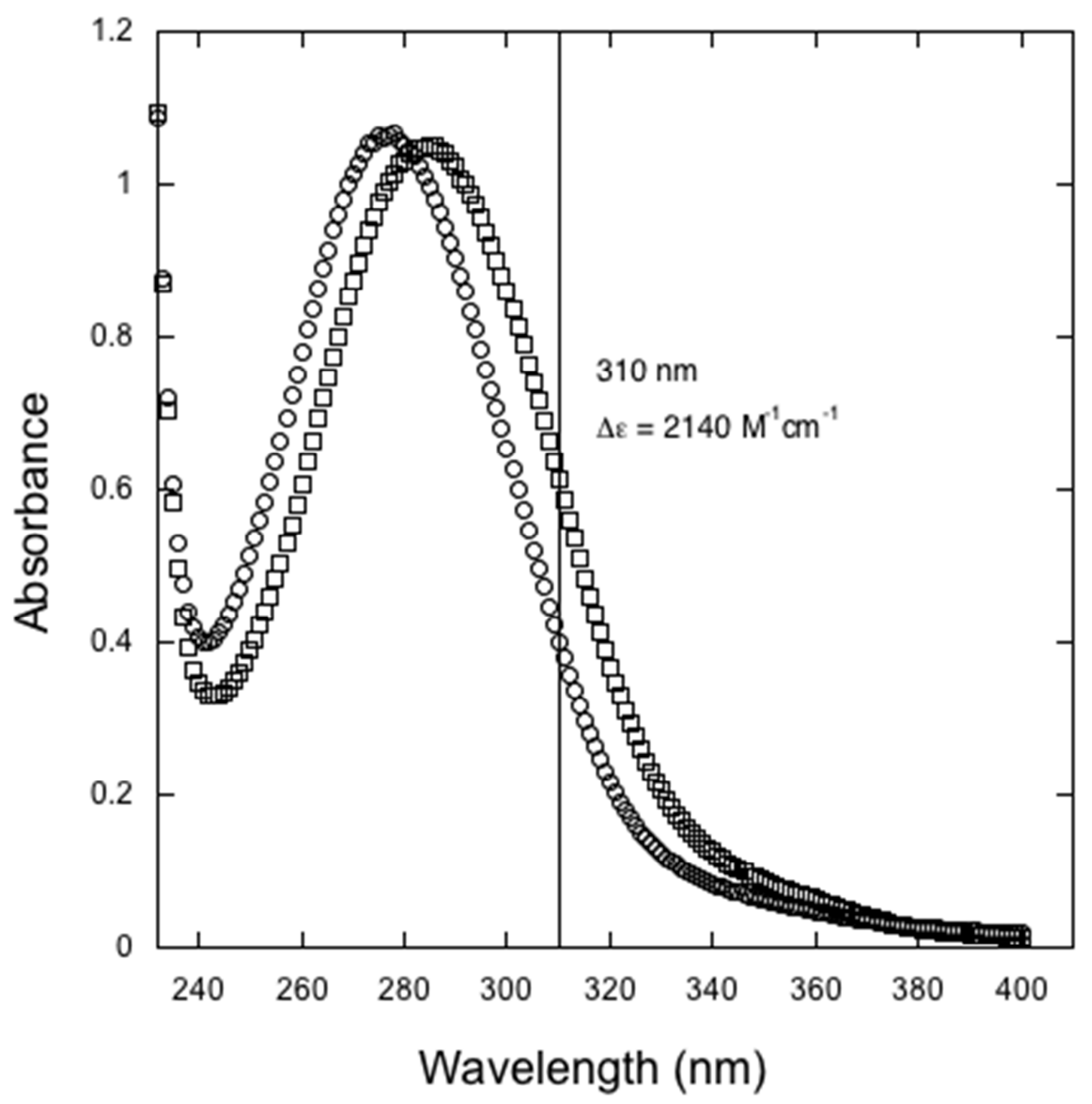

Fig. 1. Absorption spectra of $\mathbf{4}$ before $(O)$ and after $(\square)$ hydrolysis catalyzed by MAH

The methyl ester 5 was a poorer substrate than $\mathbf{4}$ because of its ten-fold higher $K_{m}$ and the ethyl ester 6 was not a substrate of MAH at all. The latter results are in accord with previous observations with mandelate esters and $\mathrm{N}$-alkyl phenylacetamides [9] which also suggested that extended leaving groups had difficulty, presumably steric, in accessing the active site. The chemically counterintuitive similar reactivity of amides and esters as substrates of MAH, 
Table 1.

Steady State Parameters for Hydrolysis of Substrates by MAH

\begin{tabular}{|c|c|c|c|}
\hline Substrate & $\mathrm{k}_{\mathrm{cat}}\left(\mathrm{s}^{-1}\right)$ & $\mathrm{K}_{\mathrm{m}}(\mu \mathrm{M})$ & $\mathrm{k}_{\mathrm{cat}} / \mathrm{Km}\left(\mu \mathrm{M}^{-1} \mathrm{~s}^{-1}\right)$ \\
\hline $\mathbf{1}^{\mathrm{a}}$ & 82 & 7.0 & 11 \\
\hline $2^{\mathrm{a}}$ & 150 & 20 & 8.3 \\
\hline $3^{\mathrm{a}}$ & 46 & 0.9 & 51 \\
\hline 4 & $43 \pm 3$ & $36 \pm 7$ & 1.2 \\
\hline 5 & $44 \pm 6$ & $400 \pm 100$ & 0.11 \\
\hline $6^{\mathrm{b}}$ & - & - & - \\
\hline $7^{\mathrm{c}}$ & 63 & 170 & 0.37 \\
\hline 8 & $5.1 \pm 0.5$ & $2300 \pm 300$ & $2.2 \times 10^{-3}$ \\
\hline $9^{d}$ & - & - & $(4.9 \pm 0.1) \times 10^{-4}$ \\
\hline 11 & $0.52 \pm 0.06$ & $470 \pm 110$ & $1.1 \times 10^{-3}$ \\
\hline 12 & $\mathrm{ND}^{\mathrm{e}}$ & ND & $(1.2 \pm 0.1) \times 10^{-5}$ \\
\hline 13 & ND & ND & $(1.7 \pm 0.1) \times 10^{-5}$ \\
\hline
\end{tabular}
a. From reference 9.
b. No reaction observed.
c. From reference 1. 
d. No sign of saturation observed at achievable concentrations $(100 \mu \mathrm{M})$.

e. ND, not determined

observed here and previously [1,9], is found also in the homologous enzyme FAAH, discussed in the introduction, and led to the proposal of a novel mechanism involving a Ser Ser Lys triad [4].

Evidence has been reported for a double displacement acyl transfer mechanism for FAAH with an acyl-enzyme intermediate located on Ser 241. Studies of the kinetics of turnover of substrate analogues suggested that acyl-enzyme formation was generally rate-limiting under substrate saturation conditions [4]. The identity of MAH as a serine hydrolase is supported by the effects of specific inhibitors. MAH, like FAAH, is inhibited for example by phenylmethylsulfonylfluoride and MAH also by the electrophilic ketone 3-phenyl-1,1,1trifluoro-2-propanone $\left(\mathrm{K}_{\mathrm{i}}=60 \mu \mathrm{M}\right)$ [1]. We have now shown that MAH is strongly inhibited by phenylmethylboronic acid $\left[\mathrm{K}_{\mathrm{a}}=(27 \pm 4) \mathrm{nM}\right]$. This compound is likely to form a tetrahedral anionic complex with the active site serine, mimicking the deacylation transition state in turnover of phenylacetamide. Boronic acids are well known to form such complexes with serine hydrolases [14]. Thus, substrates most likely react with MAH to form, initially, a covalent acylenzyme with the nucleophilic active site serine (Ser204). This mechanism is shown in Scheme 3 where the acyl-enzyme intermediate is depicted as RCOOE. This representation indicates the presence of the acyl group of normal substrates in a specific binding site (see below).

A question that does arise, however, in comparison of the ester and amide substrates, is the nature of the reaction step defining $\mathrm{k}_{\mathrm{cat}}$, acylation or deacylation of the active site serine of the enzyme (Scheme 3). Note that it is assumed in the derived equations that the second step is irreversible in the absence of added product and under initial rate conditions. It is also assumed that the reaction of the first step, substrate binding, is a fast pre-equilibrium, ie $\mathrm{k}_{-1}$, the rate constant for substrate dissociation is much greater than $\mathrm{k}_{2}$. This has also been assumed for FAAH [4]. Further, the $\mathrm{k}_{\mathrm{cat}} / \mathrm{K}_{\mathrm{m}}$ values for compounds $\mathbf{3 , 4}$, and $\mathbf{5}$ do not suggest that diffusion-controlled 
association of enzyme and substrate is rate-limiting for $\mathbf{4}$ and $\mathbf{5}$. The coincidence of $\mathbf{k}_{\text {cat }}$ values for the amide $\mathbf{4}$ and the ester $\mathbf{5}$ suggests that a common step, which would most likely be deacylation $\left(\mathrm{k}_{3}\right)$, is rate-limiting. The latter conclusion would be unusual for AS enzymes [4] but there is no fundamental reason why acylation should rate-determining for all AS enzymes and substrates. This point is reiterated below for MAH and the substrates mandelamide and methyl mandelate.

\section{Scheme 3}

$$
\begin{aligned}
& \mathrm{E} \cdot \mathrm{OH}+\mathrm{RCOX} \underset{\mathrm{K}_{5}}{\longrightarrow} \mathrm{E} \cdot \mathrm{OH} \cdot \mathrm{RCOX} \underset{\mathrm{k}_{-2}}{\stackrel{\mathrm{k}_{2}}{\longrightarrow}} \underset{+\mathrm{XH}}{\mathrm{RCOO}-\mathrm{E}} \underset{\mathrm{H}_{2} \mathrm{O}}{\stackrel{\mathrm{k}_{3}}{\longrightarrow}} \mathrm{E} \cdot \mathrm{OH}+\mathrm{RCO}_{2}^{-} \\
& k_{\text {cat }}=k_{2} k_{3} /\left(k_{2}+k_{3}\right) \quad K_{m}=K_{8}\left[k_{3} /\left(k_{2}+k_{3}\right)\right] \quad k_{c a t} / K_{m}=k_{2} / K_{8} \\
& \text { Rate-determining acylation, } k_{3}>k_{2} \\
& \mathrm{k}_{\text {cat }}=\mathrm{k}_{2} \quad \mathrm{~K}_{\mathrm{m}}=\mathrm{K}_{8} \quad \mathrm{k}_{\mathrm{cat}} / \mathrm{K}_{\mathrm{m}}=\mathrm{k}_{2} / \mathrm{K}_{8} \\
& \text { Rate-determining deacylation, } k_{2}>k_{3} \\
& k_{\text {cat }}=k_{3} \quad K_{m}=K_{s}\left(k_{3} / k_{2}\right) \quad k_{c a /} / K_{m}=k_{2} / k_{s}
\end{aligned}
$$

The values of $\mathrm{k}_{\mathrm{cat}}$ of mandelamide and methyl mandelate (7) are sufficiently different (Table 1) to preclude rate-determining deacylation at saturation for both ester and amide. Ratedetermining acylation in each case would remain possible. In order to try and resolve this issue, we decided to apply a perturbation method first described by Fitzpatrick [15]. Scheme 3 leads to the steady state equations shown in Scheme 3 it if it is assumed that substrate binding can be described as a fast equilibrium $\left(\mathrm{K}_{\mathrm{s}}\right)$.

If it is also assumed that the equilibrium constant $\mathrm{K}_{\mathrm{s}}$ is less temperature sensitive than the rate constants $k_{2}$ and $k_{3}$, then, for a given substrate, the temperature dependence of $k_{\text {cat }}$ and $\mathrm{k}_{\mathrm{cat}} / \mathrm{K}_{\mathrm{m}}$ should be very similar, reflecting $\mathrm{k}_{2}$, if acylation is rate-determining, and may be quite different if deacylation is rate-determining since the change in $\mathrm{k}_{\mathrm{cat}}$ will reflect the temperature dependence of $\mathrm{k}_{3}$ and $\mathrm{k}_{\mathrm{cat}} / \mathrm{K}_{\mathrm{m}}$ that of $\mathrm{k}_{2}$. Data from temperature dependence studies of $\mathrm{k}_{\mathrm{cat}}$ and 
$\mathrm{k}_{\mathrm{cat}} / \mathrm{K}_{\mathrm{m}}$ for $\mathbf{1}$ and $\mathbf{7}$ are shown in Figure 2 along with $\mathrm{E}_{\mathrm{a}}$ values calculated from linear fits to the data. The $\mathrm{E}_{\mathrm{a}}$ values for $\mathrm{k}_{\mathrm{cat}}$ and $\mathrm{k}_{\mathrm{cat}} / \mathrm{K}_{\mathrm{m}}$ are very different for $\mathbf{1}$ and $\mathbf{7}$ and thus, from the rationale above, deacylation is likely to be rate determining. Conversely, for $\mathbf{7}$ the $\mathrm{E}_{\mathrm{a}}$ values for $\mathrm{k}_{\mathrm{cat}}$ and $\mathrm{k}_{\mathrm{cat}} / \mathrm{K}_{\mathrm{m}}$ are the same within experimental uncertainty and thus acylation is likely to be rate determining for this substrate. It follows in the latter case that $\mathrm{K}_{\mathrm{m}}\left(=\mathrm{K}_{\mathrm{s}}\right)$ has very little temperature dependence, as required by the theory.

This result, rate-determining acylation for the ester and deacylation for the corresponding amide is chemically rather counter-intuitive because the amide is much less hydrolytically reactive than the ester. For catalysis by MAH, however, the result is reasonable since the restricted leaving group site, discussed above, may restrict enzyme acylation by the methyl ester which bears a larger leaving group than the amide. This factor is probably also involved in rationalization of the relative $\mathrm{k}_{\mathrm{cat}} / \mathrm{K}_{\mathrm{m}}$ values of $\mathbf{4}$ and $\mathbf{5}$.

As suggested by Scheme 3, hydrolysis of $\mathbf{4}$ should be impeded by addition of the protonated leaving group $(\mathrm{XH}), \mathrm{NH}_{3}$ in this case. Ammonium chloride was indeed found to be inhibitory to hydrolysis of saturating 4 with an apparent $K_{i}$ value of $(0.6 \pm 0.1) \mathrm{M}$. If it is assumed that the actual inhibitor is ammonia (Scheme 3), the corrected (assuming the pKa of ammonium to be 9.25 ) Ki value is $0.0355 \mathrm{M}$. Similarly, the hydrolysis of $\mathbf{5}$ was inhibited by methanol: $K_{i}=(0.30 \pm 0.05) M$. Thus, as required by Scheme 3, formation of the acyl-enzyme is reversible. Methylamine (to $2.0 \mathrm{M}$ ) had no effect on the rate of reaction of either $\mathbf{4}$ or $\mathbf{5}$, as expected since N-methyl-phenylacetamide is a much poorer substrate $\left(k_{\text {cat }} / K_{m}=7 \times 10^{2} \mathrm{M}^{-1} \mathrm{~s}^{-1}\right.$ [1]) than 3. 


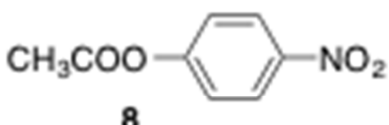

8<smiles>CC(=O)Oc1ccccc1</smiles>

9<smiles>CCCC(=O)Oc1ccc([N+](=O)[O-])cc1</smiles>

12

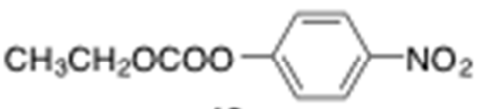

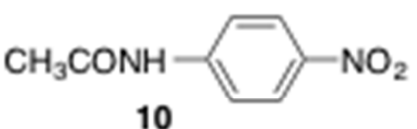

10
13 $-\mathrm{NO}_{2}$

$\mathrm{CH}_{3} \mathrm{OCOO}$<smiles>O=[N+]([O-])c1ccccc1</smiles>
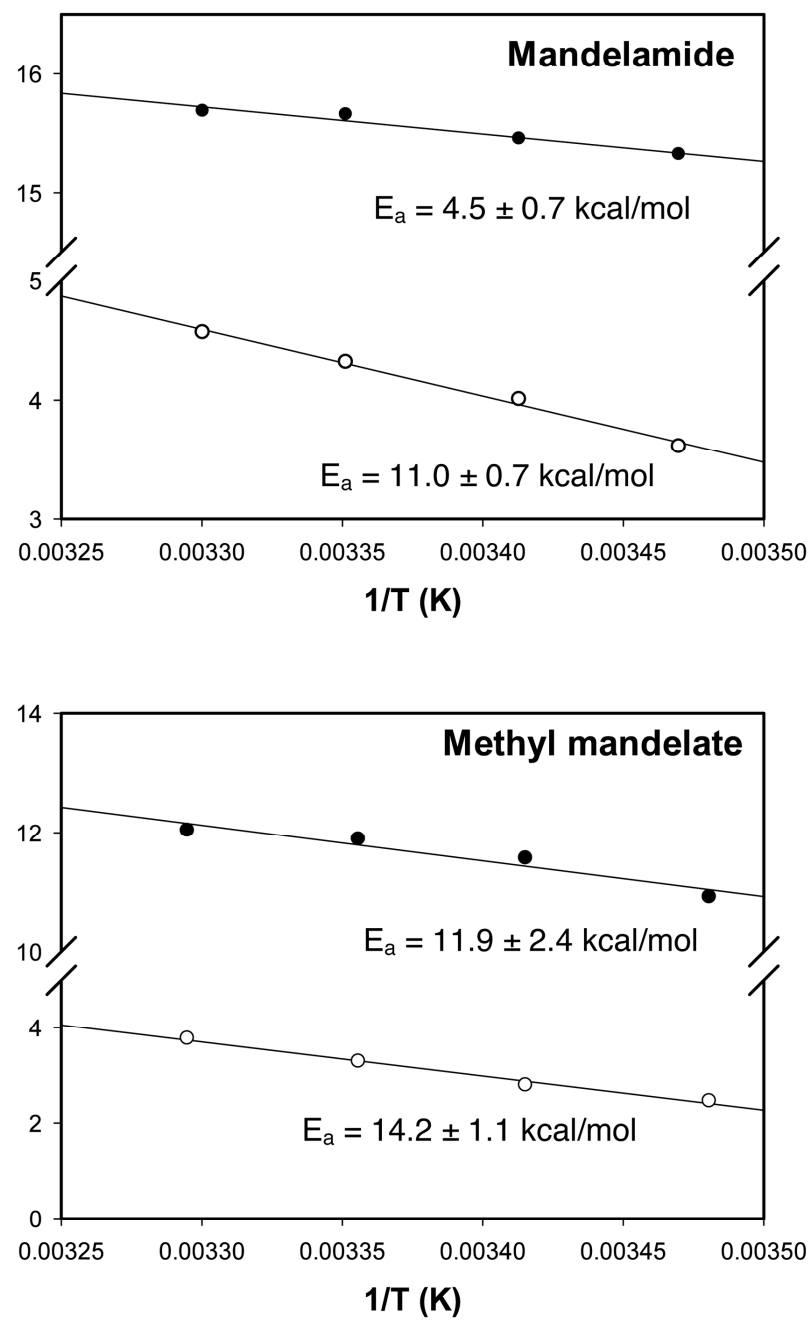
Fig. 2 Temperature dependence of $\mathrm{k}_{\mathrm{cat}}(\bullet)$ and $\mathrm{k}_{\mathrm{cat}} / \mathrm{K}_{\mathrm{m}}(\mathrm{O})$ values for hydrolysis of mandelamide and methyl mandelate catalyzed by MAH.

We have shown that MAH also catalyzes the hydrolysis of the non-specific substrate pnitrophenyl acetate $8\left[\mathrm{k}_{\text {cat }}=(5.1 \pm 0.5) \mathrm{s}^{-1}, \mathrm{~K}_{\mathrm{m}}=(2.3 \pm 0.3) \mathrm{mM}, \mathrm{k}_{\text {cat }} / \mathrm{K}_{\mathrm{m}}=2.2 \times 10^{3} \mathrm{M}^{-1} \mathrm{~s}^{-1}\right]$. It seems unlikely that the leaving group of $\mathbf{8}$, p-nitrophenolate, could fit into the leaving group site of MAH. As mentioned above, anything larger than a methoxy group does not bind there and react. In particular, p-nitrophenyl phenylacetate is not a substrate [1]. We suggest, therefore, that $\mathbf{8}$ binds and reacts in the MAH active site in reverse fashion. The p-nitrophenyl group would occupy the normal acyl site (which is quite specific for aromatic groups [1,9]) and the acetyl group would occupy the normal leaving group site. Thus there would be formed a "flipped" or inverse acyl-enzyme E-OCOCH 3 (Scheme 4), where this representation reflects occupancy by the acetyl group of the normal leaving group site.

\section{Scheme 4}
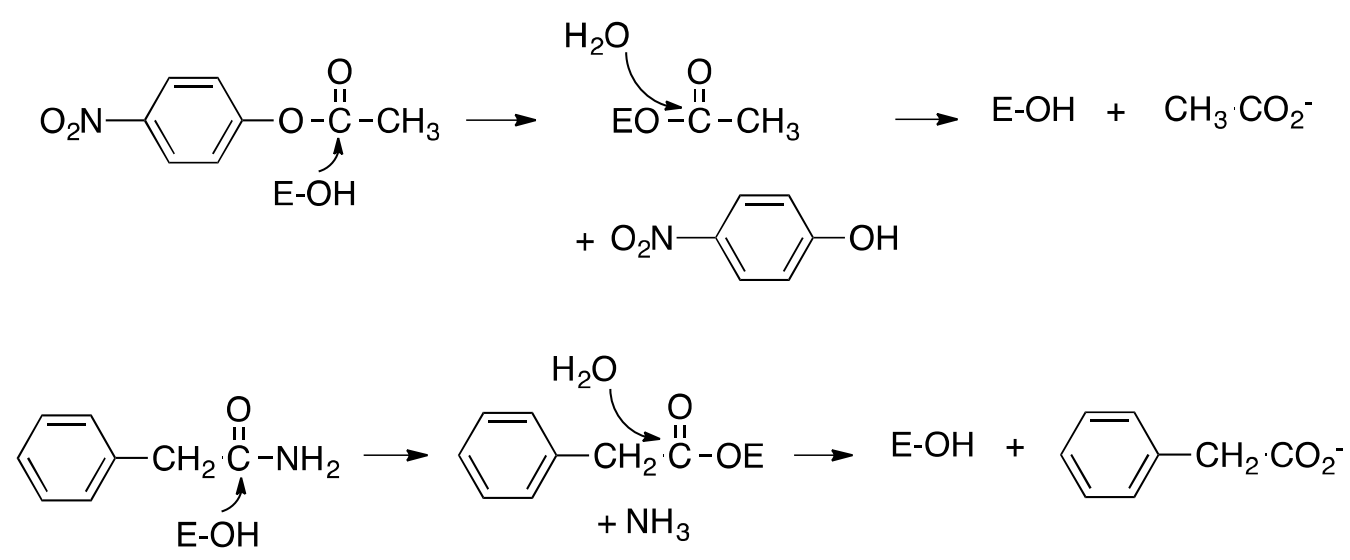

The mechanism of turnover of a normal substrate, phenylacetamide, is shown for comparison. The catalytic acids and bases also involved in catalysis have been omitted for simplicity. Unlike the normal ammonia leaving group, p-nitrophenolate would not require a general acid catalyst to leave and thus the inverse binding mode can be productive. Phenyl acetate (9) is also a substrate, 
not greatly poorer than p-nitrophenyl acetate (Table 1), suggesting that nucleophilic attack by serine rather than departure of phenolate may be the rate-determining step in acylation. The analogous amide 10, with a much poorer leaving group, is not, however, a perceptible substrate. The carbonate 11, with an acyl group still small enough to fit into the leaving group site, is also a substrate, although somewhat poorer than $\mathbf{8}$ (Table 1). The rate of hydrolysis of $\mathbf{1 1}(1.0 \mathrm{mM})$ catalyzed by MAH is not affected by addition of ammonium chloride (to $0.5 \mathrm{M}$ ). This suggests that acylation of the enzyme by 11, presumably to form $\mathrm{EOCOOCH}_{3}$, is rate determining. The same is probably true for $\mathbf{8}$ also but the ammonia experiment could not be reliably done in this case because of the higher background rate of hydrolysis and aminolysis of $\mathbf{8}$. We also note that the abnormal acyl-enzymes EO- $\mathrm{COCH}_{3}$ and $\mathrm{EO}-\mathrm{COOCH}_{3}$ may, in principle, rearrange to the normal conformation $\mathrm{CH}_{3} \mathrm{CO}-\mathrm{OE}$ and $\mathrm{CH}_{3} \mathrm{OCO}-\mathrm{OE}$ prior to hydrolysis. In this case, the kinetics and/or thermodynamics of the rearrangement may influence $\mathrm{k}_{3}$ values.

Finally, we examined the MAH-catalyzed hydrolysis of 4-nitrophenyl n-butyrate (12) and ethyl 4-nitrophenyl carbonate (13), which are analogous to $\mathbf{8}$ and $\mathbf{1 1}$, respectively, but with longer acyl groups. As expected from the above discussion, these compounds were very poor MAH substrates (Table 1) and thus, together with previous results described above, provide an accurate limit to the size of the MAH leaving group pocket (for normal substrates)

It was reported earlier that the G202A mutation of MAH strongly decreased by a factor of 20- to 50-fold the reactivity $\left(\mathrm{k}_{\mathrm{cat}} / \mathrm{K}_{\mathrm{m}}\right)$ of (acyl)aromatic substrates but that of aliphatic substrates (intrinsically weaker) increased [9]. This mutation was now found to significantly reduce $\mathrm{k}_{\mathrm{cat}} / \mathrm{K}_{\mathrm{m}}$ of $\mathbf{8}$ by a factor of 21 , a result that suggests the aromatic ring of $\mathbf{8}$ during acylation may be located at the same site as that from 3. This interpretation supports the proposal of acylation of MAH by 8 with the latter in the reverse to normal orientation. 
These experiments with $\mathbf{8}$ and $\mathbf{1 1}$ appear to open up a new avenue of reactivity of the MAH active site, which is hampered by the small leaving group cavity. This should be relevant to the design and reactivity of covalent inactivators of MAH (see below).

\subsection{Structural Interpretation}

Although a crystal structure of MAH is not available at this time, a homology model based on the crystal structure of other amidase signature enzymes, particularly FAAH, has been constructed [9]. We have used this model to interpret the substrate specificity of MAH. We constructed a model of the tetrahedral intermediate $\mathbf{1 4}$ generated from attack of MAH on the

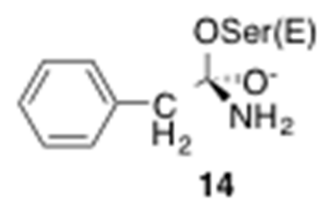

very good substrate phenylacetamide. The ligand was placed manually following the example of the crystal structure of FAAH covalently bound through its active site to a phosphonate transition state analogue (PDB ID 1MT5 [6]). After energy minimization, the structure shown in Figure 3A was obtained. This shows the substrate covalently bound to Ser204, the oxyanion $\mathrm{O}^{-}$hydrogenbonded into the oxyanion hole (NH groups of Thr201 and Gly202 and, perhaps, the side chain hydroxyl of Thr201), and the leaving group nitrogen (neutral) hydrogen bonded to the hydroxyl of Ser180, which is accompanied by Lys 100. The side chain oxygen atom of Ser 204 is also within hydrogen-bonding distance of Ser180 O $\gamma$. These placements are analogous to those in the FAAH phosphonate reference structure and can be interpreted in terms of the proposed mechanism of catalysis [4]. Two new points can be made relevant to the substrate specificity of MAH. First, the phenyl group of phenylacetamide is neatly placed in a hydrophobic cleft, stacked between the aromatic rings of Phe150 and Phe433. There is room for substitution of 
either an $\mathrm{R}$ or $\mathrm{S}$ hydroxyl group on the benzyl methylene to generate a mandelamide intermediate. The model also indicates why benzamide is not a MAH substrate [1]. In this case, the phenyl ring of benzamide would be directed, not between the phenylalanine rings, but rather directly at the phenyl ring of Phe433, leading to unfavorable steric interactions. Aliphatic amides of appropriate alkyl length, unless with bulky branched side chains [9], could also be seen as accommodating to the model.

Table 2 lists the steady state parameters for single site variants of MAH where Phe433 and Phe150 have been replaced. Mutation of Phe433, particularly to an aliphatic residue, has a large negative effect on the activity of the enzyme $\left(\mathrm{k}_{\mathrm{cat}} / \mathrm{K}_{\mathrm{m}}\right)$ but mutation of Phe150 seems to have less effect. Changes occur in both $\mathrm{k}_{\mathrm{cat}}$ and $\mathrm{K}_{\mathrm{m}}$, all unfavorable except for $\mathrm{K}_{\mathrm{m}}$ for the $\mathrm{F} 150 \mathrm{~W}$ variant which becomes smaller. The model, Figure 3A, shows the phenyl group derived from phenylacetamide closely $\pi$-stacked onto that of Phe 433 while that of Phe 150 seems to interact less closely with the substrate. The change of Phe150 to the larger Trp does seem to improve substrate binding. Thus, the results of these mutations therefore do support the model shown in Figure 3A.

Table 2. Steady state parameters for wild type MAH and certain variants (see text). The substrate was S-mandelamide in all cases.

\begin{tabular}{cccc} 
Enzyme & $\mathrm{k}_{\mathrm{cat}}\left(\mathrm{s}^{-1}\right)$ & $\mathrm{K}_{\mathrm{m}}(\mu \mathrm{M})$ & $\mathrm{k}_{\mathrm{cat}} / \mathrm{K}_{\mathrm{m}}\left(\mathrm{M}^{-1} \mathrm{~s}^{-1}\right)$ \\
\hline WT & $150 \pm 4$ & $18 \pm 2$ & $8.5 \times 10^{6}$ \\
& & & \\
F433W & $90 \pm 2$ & $43 \pm 1$ & $2.3 \times 10^{6}$ \\
& & & \\
F433L & $105 \pm 1$ & $135 \pm 5$ & $7.8 \times 10^{5}$
\end{tabular}




$$
\begin{array}{cccc}
\text { F433A } & 20 \pm 1 & 1530 \pm 30 & 1.3 \times 10^{4} \\
\text { F150W } & 43 \pm 1 & 9 \pm 1 & 4.6 \times 10^{6}
\end{array}
$$

The position of the phenyl group of phenylacetamide bound to MAH can be compared with the position of the aliphatic fatty acid side chain of a FAAH substrate. Figure 3B shows an overlap of the model of Figure 3A with the FAAH-phosphonate crystal structure mentioned above. It can be seen that just as the phenyl group of the phenylacetamide complex with MAH sits between two phenylalanine phenyl rings, the aliphatic chain of the FAAH substrate, on leaving the reaction center, lies between the hydrophobic side chains of Leu192 and Ile491 [6]. 

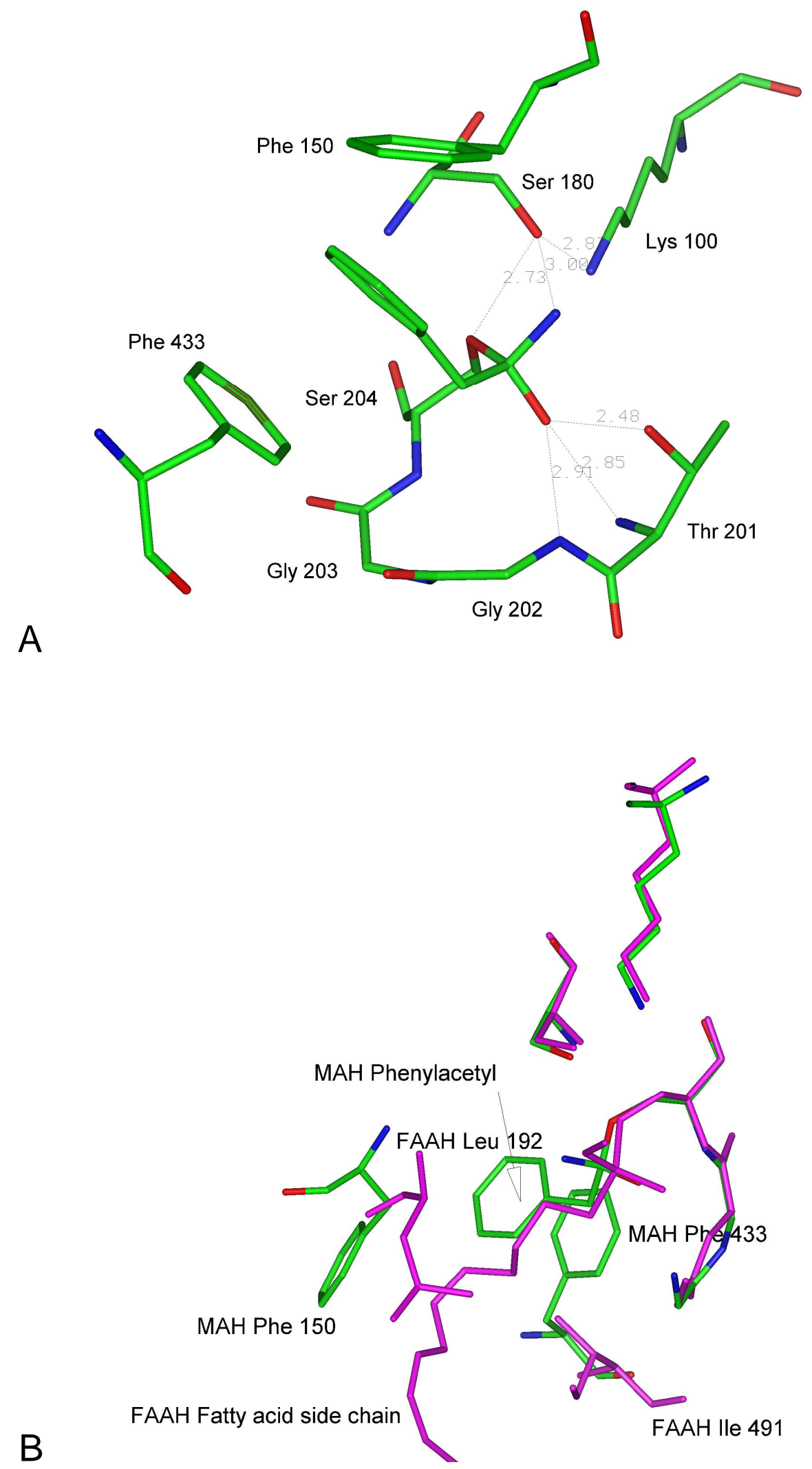
Fig 3. (A) A model of the deacylation tetrahedral intermediate derived from reaction of MAH with phenylacetamide (3) (see text). (B) Superimposition of the MAH model from above (atomic colors) and the relevant part of the crystal structure of FAAH modified by a phosphonate transition state analogue inhibitor (PDB ID 1MT5, purple). This diagram was achieved by superimposition of the $\alpha-\mathrm{C}$ of the catalytic active site residues (for MAH, these are Lys 100, Ser180, Thr201 and Ser204). 


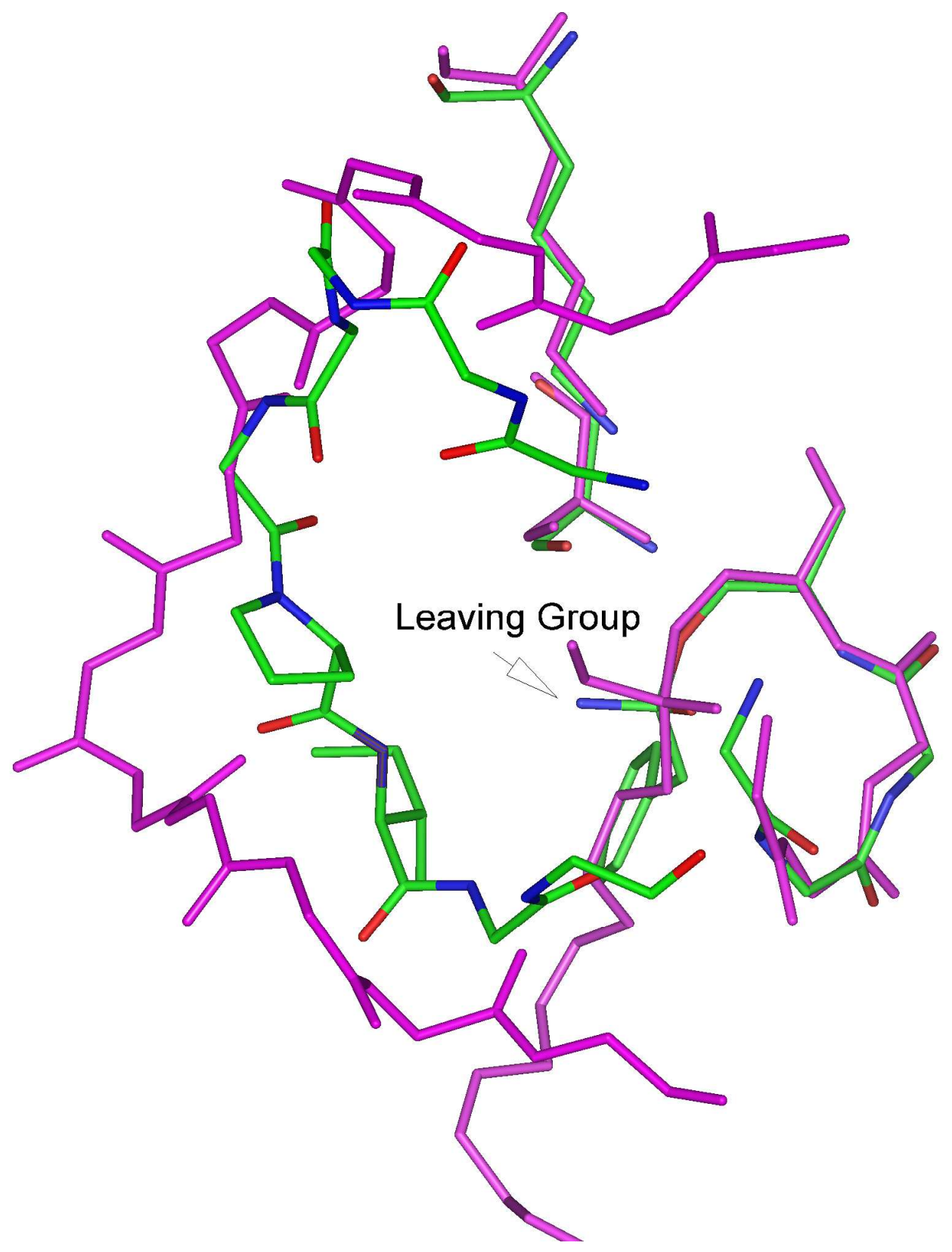

Fig 4. Extended view of the superimposition of MAH (atomic colors) and FAAH (purple) shown in Figure 2B, showing the loops adjacent to the active site in the vicinity of a leaving group during catalysis. The loops shown comprise Val226 - Pro234 (MAH) and Ser262 - Thr274 (FAAH) 
A second important aspect of MAH specificity is its strong preference for small leaving groups, with no more than two heavy atoms apparently permitted (see discussion above). MAH differs from FAAH in this respect since latter enzyme can accomodate larger substitutions in the leaving groups of substrates, e.g. anandamide [16]. The greater space in the leaving group position available to FAAH ligands has been extensively taken advantage of in the design of FAAH inhibitors such as $\alpha$-ketoheterocycles, carbamates and ureas [17]. One protein structural difference that may lead to this difference is seen in Figure 4 where relevant portions of MAH and FAAH are overlaid. A broad loop in FAAH adjacent to the leaving group position (Ser262Thr276) is replaced in MAH by a shorter one (Val226-Pro234). The loop in MAH seems well placed to unfavorably interact sterically with large leaving groups. Note that these loops are internal to the protein (the active site is buried in the protein) and not expected to be flexible [the loop in MAH did not flex significantly during a short molecular dynamic run (100 ps)].

\subsection{Inhibition by $O$-Acyl Hydroxamic Acids}

O-Acyl hydroxamic acids of general structure $\mathbf{1 5}$ are mechanistically interesting inactivators of serine $\beta$-lactamases. They act by crosslinking the active site between the nucleophilic serine and an adjacent conserved lysine [11]. The $\beta$-lactamases have active site similarity to another family of serine/lysine amidohydrolases, the $\mathrm{N}$-terminal nucleophile peptidases, which are also inhibited by $\mathbf{1 5}$ in a manner that crosslinks the active site between hydroxyl and amine groups of the N-terminal threonine/serine [8]. Since there are also congruent similarities with the active sites of amidase signature enzymes [7], we assessed the reactivity of

one of these enzymes, MAH, with several O-acyl hydroxamic acids, all distinguished from $\mathbf{1 5}$ by their small leaving groups, appropriate for MAH . Compound 16, although a primary amide, was neither a MAH substrate nor an inhibitor. The polar hydroxamate moiety in this case is apparently unable to productively engage the MAH active site. The chemically more reactive 
methyl ester analogue $\mathbf{1 7}$, however, did however react slowly to form a transient intermediate. Figure 5 shows the results of an experiment where turnover of the substrate 4 was monitored in the presence of $\mathbf{1 7}$ after addition of the enzyme to a mixture of substrate and inhibitor. The figure shows a burst of product formation from the substrate and then a slowing of turnover, presumably because of reaction of the enzyme with $\mathbf{1 7}$ to generate a transiently stable complex that then slowly breaks down to reform active enzyme. An alternative addition experiment, whereby substrate was added to a mixture of enzyme and 17, incubated for $5000 \mathrm{~s}$, yielded a largely inactive enzyme that regained activity at a rate consonant with Figure 5. The data of Figure 5 were fitted to Scheme 2 by Dynafit [13] to obtain $k_{1}$ and $k_{2}$ values (Table 3). It is likely that $\mathbf{1 7}$ yields the acyl-enzyme $\mathbf{1 8}$ as a slowly hydrolyzing intermediate since the alternative acylenzyme $\mathrm{EOCOOCH}_{3}$ hydrolyzes more rapidly (See discussion of $\mathbf{1 1}$ above). The compound $\mathbf{1 7}$ is therefore quite an effective inhibitor of MAH with an effective $K_{i}\left(=K_{m}=k_{2} / k_{1}\right)$ of $25 n$.

Unlike 16, the benzyloxy analogue 19 reacts with MAH much like 17, but acylates the enzyme much more slowly. Presumably, the more flexible side chain of $\mathbf{1 9}$ allows it weakly productive access to the active site, c.f. $\mathbf{1 6}$.

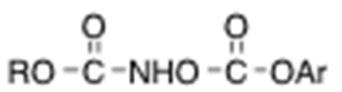

15

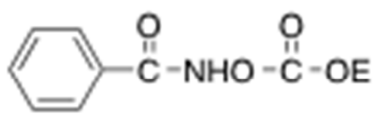

18

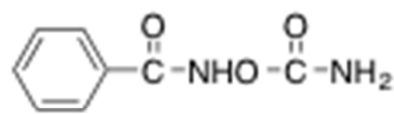

16

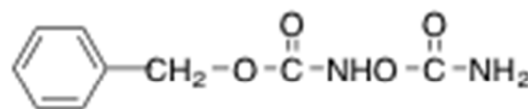

19

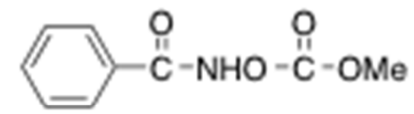

17

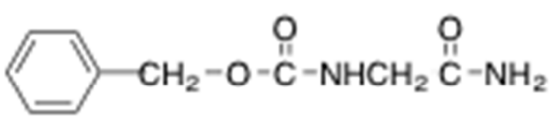

20

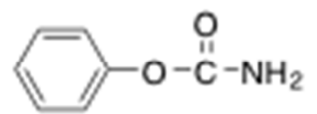

21 


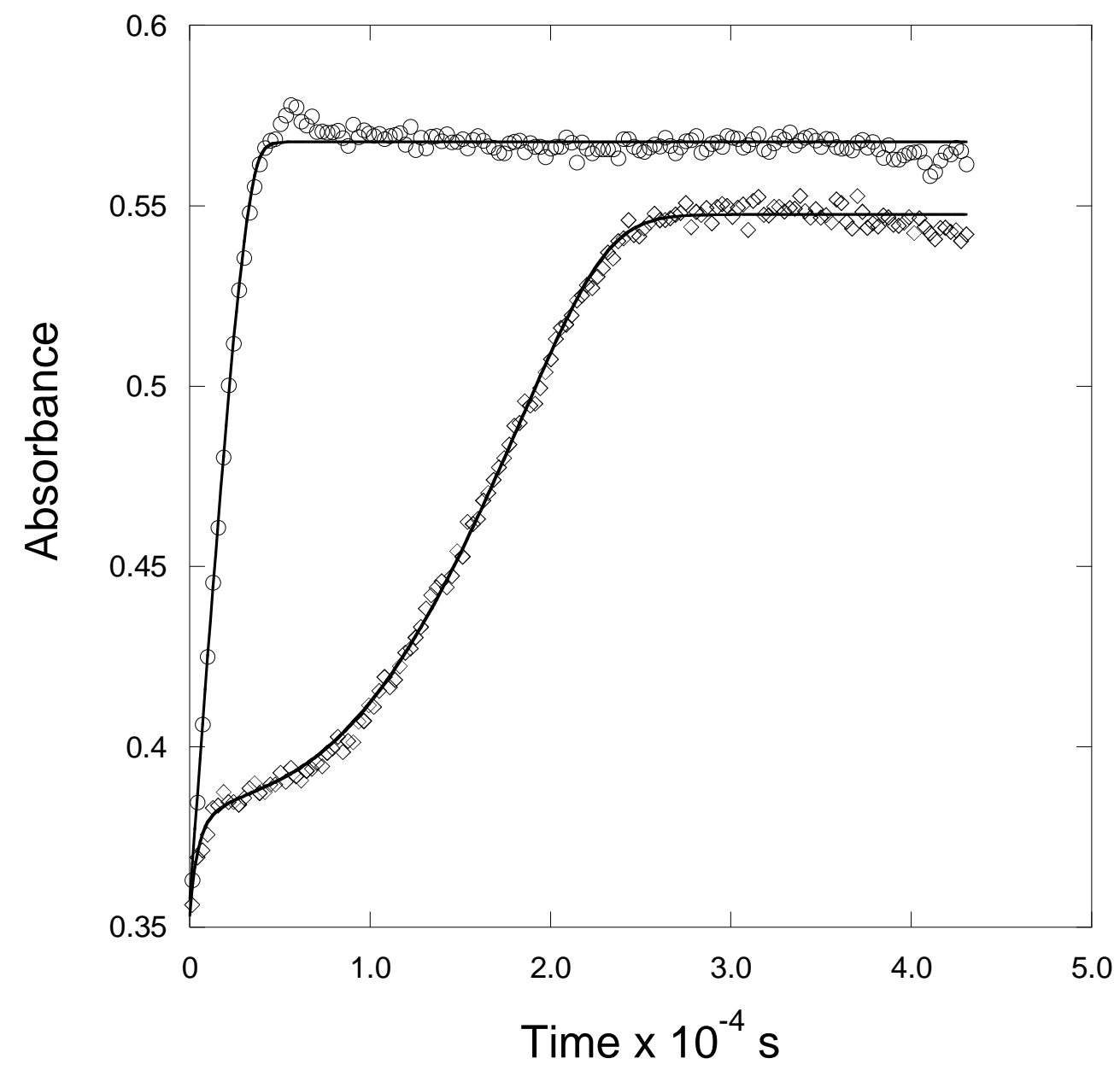

Fig 5. Turnover of $4(100 \mu \mathrm{M})$ by MAH $(14 \mathrm{nM})$ in the absence $(O)$ and in the presence $(\square)$ of $17(25 \mu \mathrm{M})$. The solid lines represent the fit of the data to Scheme 2. 


\section{Table 3.}

Kinetics of Reaction of MAH with O-Acyl Hydroxamates

$\begin{array}{ccccc}\text { Compound } & \mathrm{k}_{0} \times 10^{4}\left(\mathrm{~s}^{-1}\right) & \mathrm{k}_{1}\left(\mathrm{M}^{-1} \mathrm{~s}^{-1}\right) & \mathrm{k}_{2} \times 10^{5}\left(\mathrm{~s}^{-1}\right) & \mathrm{K}_{\mathrm{m}}\left(=\mathrm{k}_{2} / \mathrm{k}_{1}\right)(\mu \mathrm{M}) \\ \mathbf{1 6} & 7.0 & - & - & -\mathrm{a} \\ \mathbf{1 7} & 4.8 & 770 \pm 20 & 1.9 \pm 0.1 & 0.025 \\ \mathbf{1 9} & \mathrm{ND} & 0.35 \pm 0.06 & \leq 50 & \leq 1400\end{array}$

a No reaction with the enzyme observed

The question arises, however, from these results as to the reason behind the weak interactions of the amides $\mathbf{1 6}$ and $\mathbf{1 9}$ with the MAH active site. Does it derive from the fact that they are carbamates or is from the overall polarity of the side chains. These issues were examined in the following two experiments. First, the ability of N-benzyloxycarbonylglycinamide 20 (compare its structure with that of 19) to act as a substrate was tested. A ${ }^{1} \mathrm{H}$ NMR experiment showed no hydrolysis of $\mathbf{2 0}(2 \mathrm{mM})$ by MAH $(0.28 \mu \mathrm{M})$ in 3 days. Certainly, therefore, the extended polar side chain is a problem at the MAH active site. On the other hand, the presence of a carbamate also seems to present problems. Phenyl carbamate $\mathbf{2 1}$ (compare its structure with that of $\mathbf{3}$ ) was also not a MAH substrate, either by spectrophotometry or ammonia assay. The presence of the carbamate oxygen rather than a methylene group reduces $\mathrm{k}_{\mathrm{cat}} / \mathrm{K}_{\mathrm{m}}$ by at least five orders of magnitude. Clearly the carbamate is severely impeding attack by MAH, most likely because of the greater rigidity of $\mathbf{2 1}$ than $\mathbf{3}$. The carbamate moiety is planar with a rotational barrier of around $12 \mathrm{kcal} / \mathrm{mole}$ for rotation about the $\mathrm{O}-(\mathrm{C}=\mathrm{O})$ bond [18]. Molecular mechanics models suggest a rotation of $90-100^{\circ}$ around this bond, thus costing close to the maximal energy, would be needed to adjust phenyl carbamate to the ground state phenylacetamide geometry. The barrier for rotation about the $\mathrm{C}-(\mathrm{C}=\mathrm{O})$ bond of phenylacetamide is much lower. It may be then that the difficulty of achieving a reactive conformation in the 
active site is responsible for its lack of reactivity. Thus, both the polar side chain and the presence of the carbamate moiety appear to contribute to the slow reaction of O-carbamoyl hydroxamates with MAH. These compounds do not irreversibly inhibit MAH by cross-linking of the active site, as happens with $\beta$-lactamases and $\mathrm{N}$-terminal nucleophile proteases, probably because of the absence in MAH (and AS enzymes in general) of an amine directly adjacent to the nucleophilic serine hydroxyl group.

\section{Conclusions}

We have shown that p-nitrophenylacetamide is a convenient direct reading chromogenic substrate of MAH, suitable for analytical purposes. Comparison of its reactivity with MAH with that of the corresponding methyl ester confirms the comparable rates of reaction of MAH with amides and esters, similar to those previously observed with FAAH, and presumably for the same mechanistic reasons. The lack of reactivity of MAH with the corresponding ethyl ester confirms the limited size of the MAH leaving group site. Phenylmethylboronic acid, presumably a transition state analogue precursor, is a potent inhibitor of MAH. The reactivity of MAH with 4-nitrophenyl acetate (8) and 4-nitrophenyl methyl carbonate (11) suggest the formation of "inverse" acyl enzyme species where the small acyl group occupies the normal leaving group site. We have recently found that the reactivity of an N-terminal nucleophile amidohydrolase, penicillin acylase, with O-acyl hydroxamates also provides evidence for "inverted" acyl-enzyme forms [19].

There is evidence that either enzyme acylation or deacylation may be rate-determining under $\mathrm{k}_{\mathrm{cat}}$ conditions depending on the substrate. Acylation is probably rate determining for $\mathbf{1 1}$ and methyl mandelate $\mathbf{7}$ and likely for $\mathbf{8}$, while deacylation is more likely to be rate-determining for the amide 4, the ester $\mathbf{5}$ and R-mandelamide $\mathbf{1 .}$ 
We have interpreted the substrate specificity of MAH in terms of its active site structure. The aryl specificity of the acyl group of MAH substrates arises form a specific aryl binding site between two phenylalanine side chains (Phe150 and Phe433). The small leaving group site of MAH is dictated by a rigid loop of the protein backbone closely adjacent to the active site (Val226 - Pro234). These elements of specificity have been compared to those of FAAH, whose substrates have an aliphatic acyl group and the possibility of larger leaving groups.

Inactivation of MAH by O-acyl hydroxamates was slow because of the inflexible and polar hydroxamate moiety. One such compound, O-methoxycarbonylbenzohydroxamate (16), however, was a quite effective transient inactivator of MAH $\left(\mathrm{K}_{\mathrm{i}}=25 \mathrm{nM}, \mathrm{k}_{\mathrm{off}}=1.9 \times 10^{-5} \mathrm{~s}^{-1}\right)$.

\section{Conflict of interest}

The authors declare no conflict of interest.

\section{Acknowledgment}

This research was supported by Wesleyan University and by the National Science Foundation, grant EF-0425719 (MJM). 


\section{References}

[1] Gopalakrishna, K.N., Stewart, B.H., Kneen, M.M., Andricopulo, A.D., Kenyon, G.L., and Mcleish, M.J., (2004) Mandelamide hydrolase from Pseudomonas putida: Characterization of a new member of the amidase signature family. Biochemistry 43, 7725-7735.

[2] Chebrou, H., Bigey, F., Arnaud, A., and Galzy, P. (1996) Study of the amidase signature group. Biochim. Biophys. Acta 1298, 285-293

[3] McKinney, M.K. and Cravatt, B,F. (2005) Structure and function of fatty acid amide hydrolase. Annu. Rev. Biochem. 74, 411-432.

[4] Patricelli, M.P. and Cravatt, B.F. (1999) Fatty acid amide hydrolase competitively degrades bioactive amides and ester through a nonconventional catalytic mechanism. Biochemistry 38, 14125-14130.

[5] McKinney, M.K. and Cravatt, B.F. (2003) Evidence for distinct roles in catalysis for residues of the serine - serine - lysine catalytic triad of fatty acid amide hydrolase. J. Biol. Chem. 278, $37393-37399$.

[6] Bracey, M.H., Hanson, M.A., Mesuda, K.R., Stevens, R.C., and Cravatt, B.F. (2002) Structural adaptations in a membrane enzyme that terminates endocannabinoid signaling. Science 298, 1793-1796. 
[7] Pratt, R.F. and McLeish, M.J. (2010) Structural relationship between the active sites of $\beta$ lactam-recognizing and amidase signature enzymes : convergent evolution? Biochemistry 49, 9688-9697

[8] Adediran, S.A., Lin, G., Pelto, R.B. and Pratt, R.F (2012) Crossover inhibition as an indicator of convergent evolution of enzyme mechanisms : A $\beta$-lactamase and a $\mathrm{N}$-terminal nucleophile hydrolase. FEBS Lett. 586, 4186 - 4189.

[9] Wang, P.-F., Yep, A., Kenyon, G.L. and McLeish, M.J. (2009) Using directed evolution to probe the substrate specificity of mandelamide hydrolase. Protein Engin. Design Select. $22,103-110$.

[10] Rahmathullah, S.M., Hall, J.E., Bender, B.C., McCurdy, D.R., Tidwell, R.R. and Boykin, D.W. (1999) Prodrugs for amidines: synthesis and anti-Pneumocystis carinii activity of carbamates of 2,5-bis(4-amidinophenyl)furan. J. Med. Chem. 42, 3994-4000.

[11] Wyremback, P.N., Babaoglu, K., Pelto, R.B., Shoichet, B.K. and Pratt, R.F. (2007) OAryloxycarbonyl hydroxamates: new $\beta$-lactase inhibitors that cross-link the active site. $J$. Amer. Chem. Soc. 129, 9548-9549.

[12] Pelto, R. B. and Pratt R. F. (2008) Kinetics and mechanism of inhibition of a serine Blactamase by O-aryloxycarbonyl hydroxamates. Biochemistry 47, 12037-12046. 
[13] Kuzmic, P. (1996) Program DYNAFIT for the analysis of enzyme kinetic data: application to HIV proteinase. Anal. Biochem. 237, 260-273.

[14] Smoum, R., Rubinstein, A., Dembitsky, V.M. and Srebnik, M. (2012) Boron containing compounds as protease inhibitors. Chem. Rev. 112, 4156-4220.

[15] Fitzpatrick, P.F. (1989) Identification of the rate-limiting step in serine proteinases from the effect of temperature on steady-state parameters. Biochim. Biophys. Acta 995, 201-203.

[16] Cravett, B.F., Giang, D.K., Mayfield, S.P., Boger, D.L., Lerner, R.L. and Gilula, N.B. (1996) Molecular characterization of an enzyme that degrades neuromodulatory fatty acid amides. Nature, 384, 83-87.

[17] Seierstad, M. and Brietenbucher, J.G. (2008) Discovery and development of fatty acid amide hydrolase (FAAH) inhibitors. J. Med. Chem. 51, 7327-7343.

[18] Sun, H. (1993) Ab initio characterizations of molecular structures, conformation energies, and hydrogen-bonding properties for polyurethane hard segments. Macromolecules 26, 5924-5936.

[19] Adediran, S.A. and Pratt, R.F. (2017) Penicillin acylase and O-aryloxycarbonyl hydroxamates: Two acyl-enzymes, one leading to hydrolysis, the other to inactivation. Archiv. Biochem. Biophys. 614, 65-71. 
Graphical Abstract

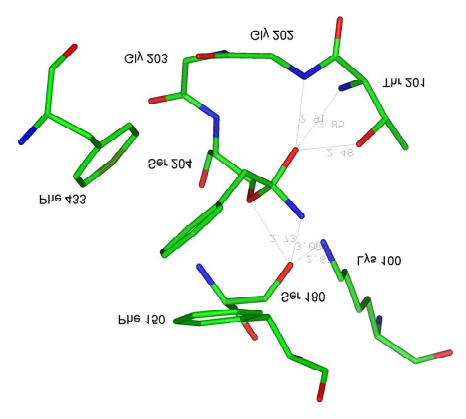

